\title{
Lean body mass change over 6 years is associated with dietary leucine intake in an older Danish population
}

\author{
Cameron Keith McDonald ${ }^{1 *}$, Mikkel Z. Ankarfeldt ${ }^{2,3}$, Sandra Capra ${ }^{1}$, Judy Bauer ${ }^{1}$, Kyle Raymond ${ }^{2}$ \\ and Berit Lilienthal Heitmann ${ }^{4,5,6}$ \\ ${ }^{1}$ Centre for Dietetic Research (C-Diet-R), University of Queensland, Brisbane, QLD 4066, Australia \\ ${ }^{2}$ Institute of Preventive Medicine, Bispebjerg and Frederiksberg Hospitals, the Capital Region, Copenhagen 2000, Denmark \\ ${ }^{3}$ Faculty of Medical and Health Sciences, University of Copenhagen, Copenhagen 2200, Denmark \\ ${ }^{4}$ Research Centre for Prevention and Health, Glostrup University Hospital, Glostrup 2600, Denmark \\ ${ }^{5}$ The Boden Institute of Obesity, Nutrition, Exercise E Eating Disorders, The University of Sydney, Sydney, NSW 2006, Australia \\ ${ }^{6}$ The National Institute of Public Health, University of Southern Denmark, Copenhagen 1353, Denmark
}

(Submitted 19 September 2015 - Final revision received 11 December 2015 - Accepted 22 January 2016 - First published online 16 March 2016)

\section{Abstract}

Higher protein intake, and particularly higher leucine intake, is associated with attenuated loss of lean body mass (LBM) over time in older individuals. Dietary leucine is thought to be a key mediator of anabolism. This study aimed to assess this relationship over 6 years among younger and older adult Danes. Dietary leucine intake was assessed at baseline and after 6 years in men and women, aged 35-65 years, participating in the Danish cohort of the WHO-MONICA (Multinational MONItoring of trends and determinants in CArdiovascular disease) study ( $n$ 368). Changes in LBM over the 6 years were measured by bioelectrical impedance using equations developed for this Danish population. The association between leucine and LBM changes was examined using multivariate linear regression and ANCOVA analyses adjusted for potential confounders. After adjustment for baseline LBM, sex, age, energy intake and physical activity, leucine intake was associated with LBM change in those older than 65 years $(n 79)$, with no effect seen in those younger than 65 years. Older participants in the highest quartile of leucine intake ( $7 \cdot 1 \mathrm{~g} / \mathrm{d})$ experienced LBM maintenance, whereas lower intakes were associated with LBM loss over 6 years (for trend: $\beta=0 \cdot 434, P=0 \cdot 03$ ). Sensitivity analysis indicated no effect modification of sex or the presence of CVD. Greater leucine intake in conjunction with adequate total protein intake was associated with long-term LBM retention in a healthy older Danish population. This study corroborates findings from laboratory investigations in relation to protein and leucine intakes and LBM change. A more diverse and larger sample is needed for confirmation of these results.

\section{Key words: Older populations: Protein intake: Nutrition: Lean body mass: Longitudinal studies}

Age-related loss of lean body mass (LBM), particularly skeletal muscle LBM, and its relationship to mortality is well cited in epidemiological studies ${ }^{(1,2)}$. Skeletal muscle LBM has been shown to decline with increasing age, and its reversal made more difficult, because of development of 'anabolic resistance', which may be related to factors such as dietary protein inadequacy $^{(3,4)}$, physical inactivity ${ }^{(5)}$, ageing vasculature $^{(6,7)}$ and chronic low-grade inflammation ${ }^{(8)}$.

Previous prospective cohort studies have shown that higher total protein intake is positively associated with measures of LBM retention ${ }^{(3,9)}$, whereas additional cross-sectional studies have reported similar ${ }^{(10)}$ as well as contrasting ${ }^{(11,12)}$ results. The most recent Nordic Nutritional Recommendations, which summarise recent key findings ${ }^{(13,14)}$, indicate that the optimal daily protein requirements are suggested as $1 \cdot 1-1.3 \mathrm{~g} / \mathrm{kg}$ body weight (BW) in elderly populations (i.e. older than 65 years) ${ }^{(15)}$. A number of smaller well-designed controlled experiments have further elucidated that increasing amounts of complete protein sources containing $2 \mathrm{~g}$ or more leucine effectively stimulate muscle protein synthesis (MPS) in elderly men ${ }^{(16)}$, with larger doses being related to a greater response ${ }^{(17,18)}$. Investigations have shown that leucine alone can signal MPS, and leucine peak, in part, can direct the amplitude of the resulting anabolic response from protein ingestion. However, in the long-term, leucine alone has been shown to have no effect on actual muscle hypertrophy over time ${ }^{(17,19)}$.

Longer-term smaller intervention studies among patients and healthy elderly ( $>6$ months) that have added a combination of leucine and the other branched-chain amino acids to normal intake have shown mixed results, with some reporting an increase in $\operatorname{LBM}^{(20,21)}$, whereas another found no significant change in LBM compared with control ${ }^{(22)}$. Therefore, accretion of skeletal LBM is most likely to be reliant upon the provision of adequate leucine in combination with a complete protein source.

Abbreviations: BF, body fat; BW, body weight; LBM, lean body mass; MONICA, Multinational MONItoring of trends and determinants in CArdiovascular disease; MPS, muscle protein synthesis.

*Corresponding author: C. K. McDonald, fax +61 73102 9207, email cam.mcdonald1@gmail.com 
It has been well established that physical activity in conjunction with the ingestion of a complete and adequate protein source yields a greater hypertrophic effect than either alone ${ }^{(16,18)}$.

Therefore, a recent position study has indicated that foods containing $2 \cdot 3-2 \cdot 8 \mathrm{~g}$ of leucine should be recommended at each meal for those over 65 years of age. In addition, a recommendation that adequate protein intake should be combined with regular aerobic and resistance training is also suggested ${ }^{(14)}$.

Although there are cross-sectional ${ }^{(10,23)}$ and prospective data $^{(3)}$ describing associations between total protein intake, LBM and LBM change, none of these studies have reported the effect on LBM in relation to dietary intake of leucine. Therefore, the purposes of this study were to investigate the relationship between dietary intake of leucine and its relationship with LBM and LBM change over 6 years and how this may differ for those younger or older than 65 years of age.

\section{Methods}

\section{Subjects}

The present study was part of the Danish MONICA (Multinational MONItoring of trends and determinants in CArdiovascular disease) project, an international study conducted under the auspices of the WHO, to monitor CVD trends and determinants. In 1982, a sample of 4807 subjects was randomly selected from the Central Person Register of all citizens born in 1922, 1932, 1942 and 1952 and living in eleven municipalities in the Copenhagen County ${ }^{(24)}$. Of these, 226 were of non-Danish origin and were excluded. Among the 4581 invited, 3608 participated in a subsequent health examination in $1982^{(25)}$. Those who participated in the baseline study (MONICA1) were invited to a follow-up examination in 1987-1988 (GEN-MONICA), of which 2987 (83\%) attended. After 11 years, 4130 of the 1982 sample were still alive and lived in Denmark. These participants were invited to the second follow-up (MONICA10) examination from 1993 to 1994, where 2554 (65\%) attended. The non-respondents have been described in detail elsewhere ${ }^{(26)}$.

Among survivors of the participants of MONICA1, one in six individuals was invited for a diet history interview at GEN-MONICA and MONICA10 surveys conducted 6 and 11 years later ( $n$ 552). Body composition measures were collected only at the GEN-MONICA and MONICA10 surveys, and total information on both dietary and body composition data for both GEN-MONICA and MONICA10 was available for 368 participants. Of those, leucine intake at MONICA1 was also available for 233 participants. For the purposes of this study, data from only GEN-MONICA and MONICA10 will be used. These two surveys will be referred to as 'baseline' and 'follow-up', respectively.

The present study was conducted according to the guidelines laid down in the Declaration of Helsinki (1989) of the World Medical Association, and the Ethics Committee of the Medicine Faculty of the Copenhagen County approved all the procedures involving human subjects. All subjects were informed of the aims and procedures of the study, and they gave their written consent

\section{Anthropometry}

BW was measured to the nearest $0.1 \mathrm{~kg}$ using a lever balance, with subjects dressed in light clothing or underwear. Height was measured without shoes to the nearest $0.5 \mathrm{~cm}$. A bioelectrical impedance (BIA)-103 body-composition analyzer (RJL Systems) was used to measure electrical impedance, following the manufacturer's instructions. Measurements were taken with a tetra polar electrode arrangement while the subjects were lying relaxed on a couch. The electrodes were placed on the dorsal surfaces of the right hand and foot at the distal metacarpals and metatarsals, respectively, and between the distal prominence of the radius and the ulna at the wrist and the medial and lateral malleoli at the ankle. A specific equation for estimating body fat (BF) from impedance, with measurements of total body water and $\mathrm{K}$ as a reference, has been developed specifically for this population and reported previously ${ }^{(16)}$ :

$$
\begin{aligned}
\mathrm{BF}(\mathrm{kg})= & 0 \cdot 819 \mathrm{BW}(\mathrm{kg})-0 \cdot 279 \mathrm{Ht}^{2} / R \\
& \left(\mathrm{~cm}^{2} / \Omega\right)-0.064 \mathrm{sex} \times \mathrm{BW}(\mathrm{kg}) \\
& +0 \cdot 077 \text { age }(\text { years })-0.231 \mathrm{Ht}(\mathrm{cm}) \\
& +14.941 .
\end{aligned}
$$

where $\mathrm{BF}$ is the body fat, $\mathrm{Ht}$ the height and BW the body weight. Sex was coded as 1 for men and 0 for women. LBM (kg) was calculated by subtracting BF ( $\mathrm{kg}$ ) from total BW $(\mathrm{kg})$.

$\mathrm{LBM}$ and $\mathrm{BF}$ measures were available at baseline and follow-up surveys, but unavailable at MONICA1. Thus, our analysis focused on change in LBM between these two latter time points.

\section{Dietary assessment}

At baseline and follow-up, the same dietitian interviewed participants to assess dietary intake based on information from the previous month, and average daily nutrient and energy intakes were calculated from this information. Meal patterns, dishes and foods were explored by interview using a detailed pre-coded interview form. Quantities were explored using food models, photo series, cups and measures. This instrument has been validated in another study ${ }^{(27)}$. Nutrient calculations have been carried out with the DANKOST programme, which is derived from the Danish food composition tables. This database included 835 food items at baseline in 1988, and it was extended and comprised 1250 food items in 1995. In 1987-1988, participants gave information on diet intake based on 498 different foods, and at follow-up in 1993-1994 1177 were recorded. Food contents of leucine and all the other amino acids were supplied from the food database, which can be found at www.foodcomp.dk.

\section{Physical activity}

Leisure-time activity was based on participants marking one out of four alternatives: (i) almost completely inactive, reading, television viewing, cinema-going; (ii) some physical activity (at least $4 \mathrm{~h} /$ week); (iii) regular activity; and (iv) regular, hard 
physical training for competition. These variables were then reduced by restricting sedentary activity to (i) only and combining (ii) + (iii) + (iv) as the physically active group.

\section{Demographical variables}

Participants recorded present and previous smoking habits and were classified as either current smokers or non-current smokers. Women recorded whether they had entered menopause. Information on previous or prevalent disease, including CVD and cancer diagnoses, was obtained by following-up each individual via the unique personal number in the centralised hospital discharge registry, which is complete with information for all individuals hospitalised in Denmark since 1973.

\section{Statistical analysis}

Differences in demographical data between older ( $>65$ years of age) and younger ( $<65$ years of age) participants at baseline were assessed using Mann-Whitney $U$ and $\chi^{2}$ tests for continuous and categorical variables, respectively.

Multivariable linear regressions and ANCOVA were used to assess the relationship between leucine at baseline in 1987-1988 and LBM change until 1993-1994, when leucine was expressed as a continuous variable and categorical (quartiles) variable, respectively. The covariates included in the primary model were LBM (kg), BW (kg) and physical activity at baseline, sex and age. Physical activity was reduced to a binary variable by dividing the groups as (i) and (ii) + (iii) +(iv)
Interaction product and terms were used in the linear regression for sensitivity analyses. Sex, physical activity, and presence of CVD were assessed for their interaction on the relationship between leucine and LBM. For interactions that approached significance $(P<0 \cdot 1)$, stratified analyses were carried out to determine their influence on the overall model.

\section{Results}

For the 368 subjects who had diet information and complete data on changes in body composition between assessments at baseline in 1987-1988 and follow-up in 1993-1994, the mean age was $50 \cdot 4$ ( $\mathrm{sD} 10 \cdot 8$ ) years, $51 \cdot 1 \%$ were female and all were Caucasian (Table 1). The mean daily leucine intake was significantly greater for those aged 35-55 years compared with those aged $>65$ years. In addition, those aged $>65$ years had significantly lower LBM, total energy and protein intakes, higher BF percentage and BMI, and were less likely to have never smoked. For the whole population, there was a mean LBM loss, which was significantly greater for those aged $>65$ years compared with those aged $<65$ years.

\section{Dietary leucine intake and subsequent lean body mass change over 6 years}

After adjustment for sex, age, total energy intake, physical activity and LBM at baseline, no significant associations were found between baseline leucine intake and the subsequent 6 -year LBM change for all individuals $(\beta=0.000, P=0 \cdot 99)$. For

Table 1. Baseline demographics

(Numbers and percentages; mean values and standard deviations; mean values with their standard errors)

\begin{tabular}{|c|c|c|c|c|c|c|c|}
\hline & \multicolumn{2}{|c|}{ Overall ( $n$ 368) } & \multicolumn{2}{|c|}{ Age <65 years $(n$ 289) } & \multicolumn{2}{|c|}{ Age $>65$ years $(n 79)$} & \multirow[b]{2}{*}{$P^{\star}$} \\
\hline & Mean & SD & Mean & SD & Mean & SD & \\
\hline \multicolumn{7}{|l|}{ Female } & NS† \\
\hline$n$ & \multirow{2}{*}{\multicolumn{2}{|c|}{$\begin{array}{l}188 \\
51.1\end{array}$}} & \multirow{2}{*}{\multicolumn{2}{|c|}{$\begin{array}{c}148 \\
51.2\end{array}$}} & \multirow{2}{*}{\multicolumn{2}{|c|}{$\begin{array}{l}40 \\
50.6\end{array}$}} & \\
\hline$\%$ & & & & & & & \\
\hline Age (years) & $50 \cdot 4$ & $10 \cdot 8$ & $46 \cdot 1$ & 8.0 & $65 \cdot 7$ & 0.04 & 0.000 \\
\hline Body weight $(\mathrm{kg})$ & $69 \cdot 6$ & 0.88 & 68.7 & 1.01 & $70 \cdot 0$ & 0.75 & 0.6 \\
\hline $\mathrm{BMI}\left(\mathrm{kg} / \mathrm{m}^{2}\right)$ & $24 \cdot 2$ & 0.23 & 24 & 0.27 & $25 \cdot 2$ & 0.46 & 0.001 \\
\hline LBM $(\mathrm{kg})$ & & & & & & & 0.007 \\
\hline Mean & \multirow{2}{*}{\multicolumn{2}{|c|}{$\begin{array}{c}49.2 \\
0.66\end{array}$}} & \multirow{2}{*}{\multicolumn{2}{|c|}{$\begin{array}{r}51.8 \\
0.5\end{array}$}} & \multirow{2}{*}{\multicolumn{2}{|c|}{$\begin{array}{c}48.6 \\
1.31\end{array}$}} & \\
\hline $\mathrm{SE}$ & & & & & & & \\
\hline $\mathrm{BF} \%$ & $27 \cdot 1$ & 0.5 & $26 \cdot 5$ & 0.57 & 31.6 & 0.91 & 0.000 \\
\hline Change in LBM (5 year) (kg) & -0.075 & 1.7 & 0.04 & 1.7 & -0.67 & 0.09 & 0.008 \\
\hline Total daily energy intake $(\mathrm{MJ} / \mathrm{d})$ & 8.8 & 2.9 & 9.0 & 3.0 & 8.7 & 0.39 & 0.02 \\
\hline Total daily protein intake $(\mathrm{g} / \mathrm{d})$ & 74.6 & 1.5 & $77 \cdot 8$ & 1.22 & 68.9 & 2.5 & 0.008 \\
\hline \multirow[t]{2}{*}{ Total daily Leu intake $(\mathrm{g} / \mathrm{d})$} & $5 \cdot 47$ & 0.12 & 5.7 & 0.14 & $5 \cdot 3$ & 0.081 & 0.004 \\
\hline & $n$ & $\%$ & $n$ & $\%$ & $n$ & $\%$ & \\
\hline \multicolumn{8}{|l|}{ Lifetime prevalence of disease } \\
\hline CVD diagnosis (at baseline) & 30 & 8.2 & 21 & $7 \cdot 3$ & 9 & 11.4 & $0.03+$ \\
\hline Anytime (until 1994) & 41 & $11 \cdot 1$ & 27 & $9 \cdot 3$ & 14 & $17 \cdot 7$ & $0.04 \dagger$ \\
\hline \multicolumn{8}{|l|}{ Physical activity leisure } \\
\hline Sedentary & 84 & $22 \cdot 8$ & 70 & $24 \cdot 2$ & 14 & $17 \cdot 7$ & NS† \\
\hline Physically active & 284 & $77 \cdot 2$ & 219 & $75 \cdot 8$ & 65 & $82 \cdot 3$ & NSt \\
\hline
\end{tabular}

LBM, lean body mass; BF\%, body fat percentage.

${ }^{*} P$ value comparing those over 65 years of age with those younger than 65 years.

$\dagger x^{2}$ Test, all others Mann-Whitney $U$ tests with the grouping $>65$ or $<65$ years of age; sedentary: almost completely inactive: reading, television viewing, cinema-going; physically active: some physical activity (at least $4 \mathrm{~h} /$ week) or more. 
those aged $>65$ years at baseline, a linear relationship was found for leucine intake as a continuous variable $(\beta=0.434$, $P=0.028$ ). Fig. 1 shows results of the adjusted ANCOVA for LBM changes by quartiles of leucine intake, which indicated that only those in highest quartile of leucine intake $(7 \cdot 1(\mathrm{SD} 0 \cdot 6) \mathrm{g} / \mathrm{d})$ maintained LBM (adjusted mean change in LBM: $0.24 \mathrm{~kg} ; \quad 95 \%$ CI $-0.67,1.14)$. The lower three quartiles experienced loss of LBM over time (Q1: $-1.24 \mathrm{~kg}$; 95\% CI -1.9, -0.5; Q2: -0.99 kg; 95\% CI -0.88, 0.5; Q3: -0.2 kg; $95 \%$ CI $-0.88,0.5)$. For those aged $<65$ years at baseline, no association was found between leucine intake as a continuous variable and change in $\operatorname{LBM}(\beta=-0.082, P=0.39)$. In addition, no differences were seen between any of the quartile groups (Q1: $0.01 \mathrm{~kg}$; 95\% CI -0.5, 0.5; Q2: $-0.06 \mathrm{~kg}$; 95\% CI -0.5, 0.4; Q3: 0.4 kg; $95 \%$ CI 0.0, 0.8; Q4: $-0 \cdot 1 \mathrm{~kg} ; 95 \%$ CI $-0 \cdot 3,+0 \cdot 1$. Table 2 shows total protein and leucine intakes across the four quartiles of intake and the corresponding change in LBM for younger and older individuals. Compared with those older than 65 years, relative total protein and leucine intakes were higher in all four corresponding quartiles for those younger than 65 years.

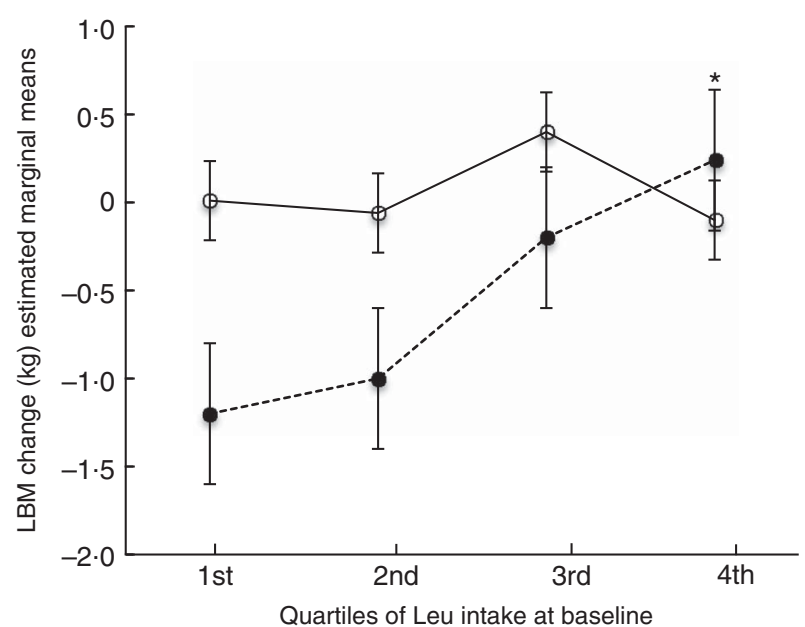

Fig. 1. Six-year lean body mass (LBM) change for quartiles of leucine intake. ${ }^{\star} P=<0.05$. ......... Relates to quartile values for those $>65$ years of age (for trend as a continuous variable: $\beta=0.43, P=0.028) ;-$ relates to those $<65$ years of age (for trend: $\beta=-0.08, P=0.393$ ). Adjusted model for ANCOVA includes LBM at baseline, total energy intake, age, sex and physical activity.
Sensitivity analyses were performed that included interactions with history of CVD diagnosis at baseline and/or follow-up, sex or physical activity. For those older than 65 years, a significant interaction was found for 'any diagnosis of CVD' (i.e. diagnosed with CVD at baseline or follow-up) $\times$ leucine $(\beta$ : -1.031 , $P=0 \cdot 008$ ), whereas no interaction was seen for younger individuals $(\beta: 0 \cdot 199, P=0 \cdot 25)$. After restricting the analysis to only older individuals free of CVD, a significant association for leucine and change in LBM $(\beta=0.481, P=0.04 ; n$ 65) was seen. However, for those over 65 years and with 'any diagnosis of CVD', no relationship was found between leucine and LBM change $(\beta=-0 \cdot 171, P=0 \cdot 76 ; n$ 14). Post hoc analyses revealed that those free of CVD ( $n$ 65) lost less LBM than those with any diagnosis of CVD ( $n$ 14) over 6 years (mean change: -0.35 (SD 1.4) $v .-1 \cdot 3(\mathrm{SD} 1 \cdot 8) \mathrm{kg}$, respectively; $t=2 \cdot 2, P=0 \cdot 03$ ).

For those younger than 65 years, a significant interaction was found by level of physical activity $(P=0.03)$. However, when analyses were restricted to those who were sedentary $(P=0 \cdot 28$; $n$ 69) or physically active $(P=0.065 ; n$ 217), no significant associations were found between leucine and LBM change. No statistically significant interaction for leucine $\times$ physical activity was seen in older individuals. A trend for sex $\times$ leucine interaction was noted in older individuals $(\beta: 0.72, P=0 \cdot 11)$. However, no significant trends were noted when analyses were restricted to males $(\beta: 0 \cdot 445, P=0 \cdot 10)$ or females only $(\beta: 0.385$, $P=0 \cdot 14)$.

\section{Discussion}

In a healthy population subset of adult Danes, higher dietary protein and leucine intakes were associated with greater LBM retention in those aged 65 years, whereas no association was found for those younger than 65 years. For those aged $>65$ years, LBM was maintained over 6 years in individuals who consumed a mean of $7 \cdot 1 \mathrm{~g}$ of leucine/d, with a corresponding mean total protein intake of $1.25 \mathrm{~g} / \mathrm{kg} \mathrm{BW}$ per $\mathrm{d}$. Therefore, a long-term relationship between leucine intake in the presence of adequate total protein consumption and LBM retention was shown. This association remained significant after adjustment for initial LBM, total energy intake, age, sex, physical activity, smoking status and presence of CVD.

The presence of a diagnosis of CVD at any time during the 6-year follow-up period was associated with greater LBM loss,

Table 2. Quartiles of total leucine and corresponding relative total protein intakes for those younger and older than 65 years of age (Mean values and standard deviations)

\begin{tabular}{|c|c|c|c|c|c|c|c|c|}
\hline & \multicolumn{2}{|c|}{ Quartile 1} & \multicolumn{2}{|c|}{ Quartile 2} & \multicolumn{2}{|c|}{ Quartile 3} & \multicolumn{2}{|c|}{ Quartile 4} \\
\hline & Mean & SD & Mean & SD & Mean & SD & Mean & SD \\
\hline \multicolumn{9}{|c|}{ Protein intake ( $\mathrm{g} / \mathrm{kg} \mathrm{BW}$ per d) } \\
\hline$<65$ years & 0.75 & 0.2 & 0.94 & 0.2 & $1 \cdot 10$ & 0.2 & 1.40 & 0.4 \\
\hline$>65$ years $^{*}$ & 0.61 & 0.2 & 0.92 & 0.1 & 0.97 & 0.2 & 1.26 & 0.2 \\
\hline \multicolumn{9}{|l|}{ Leu intake $(g / d)$} \\
\hline$<65$ years & $3 \cdot 67$ & 0.6 & 4.80 & 0.25 & 5.88 & 0.35 & 8.08 & 1.5 \\
\hline$>65$ years ${ }^{\star}$ & 3.03 & 0.9 & 4.44 & 0.3 & 5.56 & 0.4 & $7 \cdot 12$ & 0.6 \\
\hline
\end{tabular}

BW, body weight.

* Compared with those $<65$ years, those aged $>65$ years had significantly lower intakes of protein and leucine for all quartiles $(P<0.05)$. 
and similarly in CVD-free individuals, leucine and total protein intakes had a more marked influence over LBM. Individuals with a diagnosis of CVD may have been exposed to greater levels of inflammation that may have increased anabolic resistance in them ${ }^{(8)}$, which in turn may have dampened the effect of anabolic stimuli including the muscle protein synthetic effects of protein intake. Alternatively, as previous case-control studies in Nordic countries have indicated that those with CVD reported higher-quality diets than healthy controls at the time after diagnosis ${ }^{(28)}$, it is plausible to suggest that those diagnosed with CVD may have altered their dietary intake rendering their baseline data on diet intake unreliable, which may explain why the relationship between leucine and LBM change was strengthened when including only those without CVD.

Previous observational research findings from the Health, Ageing and Body Composition (Health ABC) study have indicated that individuals in the lowest quintile of protein intake $(0.7 \mathrm{~g} / \mathrm{kg}$ BW) experienced a $40 \%$ greater loss of LBM over 3 years compared with the highest quintile $(1-1.2 \mathrm{~g} / \mathrm{kg} \mathrm{BW})^{(3)}$. Similar observations were made in a Chinese sample assessing LBM change by mid-arm circumference ${ }^{(9)}$. In addition, protein intakes from animal meats, which typically contain the highest concentrations of leucine ${ }^{(29)}$, have been associated with greater LBM retention compared with vegetable-based protein $^{(3,23)}$. Laboratory data in younger and older individuals have indicated that after a meal containing $2 \mathrm{~g}$ of leucine in the form of a complete whey protein isolate ${ }^{(16)}$, or after $170 \mathrm{~g}$ of meat ${ }^{(30)}$, MPS is increased, resulting in net LBM gain. More in-depth investigations have shown that for those over 65 years, a positive dose-response relationship between protein ingestion and MPS exists for up to $40 \mathrm{~g}$ of protein (containing $4 \mathrm{~g}$ of leucine). However, younger individuals experienced no further gains after $20 \mathrm{~g}$ of leucine (containing $2 \mathrm{~g}$ leucine) at one meal ${ }^{(31)}$. To frame these findings correctly, it is important to note that free leucine added to an adequate and complete protein source does not provide further anabolic advantages ${ }^{(32)}$. Thus, leucine is an essential factor and a useful surrogate marker for determining effect on MPS, yet total essential amino acid intake will ultimately determine the anabolic effect of protein ingestion.

Combining both bodies of literature, a recently published position stand concluded that protein sources containing $2 \cdot 3-2 \cdot 8 \mathrm{~g}$ of leucine are recommended at each main meal (total intake of $6 \cdot 6-8 \cdot 4 \mathrm{~g} / \mathrm{d}$ ) to optimise LBM outcomes in older populations ( $>65$ years) ${ }^{(14)}$. Our study is the first to confirm both observational and experimental data with regard to LBM and leucine intake. Furthermore, our results confirmed that protein consumed through foods without protein supplementation seems sufficient to maintain LBM for a longer term in older populations.

In the present study, there was no association between protein intake and LBM change for those younger than 65 years. From laboratory data, it is known that older individuals need more protein than younger individuals in order to elicit equivalent responses in $\mathrm{MPS}^{(16)}$. Therefore, declines in anabolic response $^{(33,34)}$ in older individuals may partly explain why higher protein intake may be required for clinically relevant LBM preservation, than for those younger than 65 years. Similarly, markers of chronic inflammation are known to contribute to age-related sarcopenia $^{(8)}$ and are predictive of $\mathrm{CVD}^{(35)}$. No statistically significant interactions were seen overall for sex $\times$ leucine; however, a trend for interaction was noted for older but not younger individuals. Previous literature suggests that women may have a higher rate of basal MPS ${ }^{(36,37)}$; however, one study indicated a similar MPS response to feeding in older men and women (65-80 years) ${ }^{(37)}$. Our findings agree with one previous study that reported no differences between sexes in middle-aged men and women in either basal or fed states ${ }^{(38)}$. However, a greater sample size with more detailed physical activity data may elucidate sex differences in future studies. No interaction was noted for physical activity $\times$ leucine. The current literature indicates that resistance training and leucine intake combined produce greater MPS responses and LBM gains compared with either intervention alone ${ }^{(18)}$. In addition, a cross-sectional study reported greater skeletal muscle LBM for those having higher protein intakes in conjunction with both vigorous aerobic training or strength training ${ }^{(10)}$. Our physical activity classification of 'sedentary' or 'non-sedentary' did not delineate aerobic and resistance exercise, thus potentially explaining the lack of effect seen in the previous literature.

A few limitations should be noted. It is possible that assessment of dietary intake of leucine at baseline may have been inadequate to capture important dietary exposures over the subsequent 6 years; however, the power was adequate to detect a linear relationship. Furthermore, we showed previously that fat- and/or carbohydrate-rich foods are most under-reported, and protein intake is also under-reported in general and particularly among the more obese ${ }^{(39)}$. Therefore, both random reporting error, which would tend to attenuate, and non-random reporting error, which may have inflated the observed association, are possible in the present study ${ }^{(39)}$. The net effect of such opposite acting errors is unknown. However, LBM retention in older individuals occurred at similar amounts protein and leucine previously reported to elicit net LBM growth in both laboratory and epidemiological studies ${ }^{(14)}$. In addition, the reliability of our results related to measurement of LBM was strengthened by using a population-specific algorithm generated from the measurement of total body water and total $\mathrm{K}$ as reference ${ }^{(40)}$. Our entire sample included Danes of Caucasian descent; thus, our results are most likely valid for Caucasian populations and will require confirmation in other races. In contrast, the use of BIA as the method of body composition assessment is likely to be less accurate than a more current gold standard measure using dual-energy X-ray absorptiometry or computer tomography scan ${ }^{(41)}$. However, the use of a population-specific BIA algorithm used for this trial specifically is a factor that may have improved the precision of these measures. Finally, resistance training has been the most common type of exercise stimulus for measurement of anabolic response, and data for resistance training were not available.

\section{Conclusion}

For Danes aged 65 years, greater dietary intake of leucine is associated with greater retention of LBM over 6 years. These results confirm previous observational and laboratory findings that to avoid loss of LBM in older populations a mean intake of $1.25 \mathrm{~g}$ of protein $/ \mathrm{kg}$ BW per $\mathrm{d}$ containing $7 \cdot 1 \mathrm{~g} / \mathrm{d}$ of leucine 
(approximately $2 \cdot 3-2.4 \mathrm{~g} /$ main meal) may be required. These findings should be confirmed in a larger and more diverse population.

\section{Acknowledgements}

The authors thank the participants in the Danish cohort of the WHO MONICA trial.

Funding for travel to perform the research was provided by the University of Queensland (UQ) through the Graduate Student International Travel Award (GSITA). Research and statistical analysis support was provided by the Institute of Preventive Medicine, Bispebjerg and Frederiksberg Hospitals. This research was supported by the UQ GSITA and by a Danish MRC grant from the FREJA programme (Female Researchers in Joint Action).

The authors' responsibilities were as follows - C. K. M.: analysis, drafting of the manuscript; M. Z. A., S. C., J. B. and B. L. H.: critical revision of the manuscript; K. R.: data extraction, translation and design of data analysis; C. K. M. and B. L. H.: study design; B. L. H.: collection of data; C. K. M., M. Z. A. and B. L. H.: interpretation of data. All authors read and approved the final manuscript.

The authors declare that there are no conflicts of interest.

\section{References}

1. Bunout D, de la Maza MP, Barrera G, et al. (2011) Association between sarcopenia and mortality in healthy older people. Australas J Ageing 30, 89-92.

2. Muscaritoli M, Anker SD, Argilés J, et al. (2010)) Consensus definition of sarcopenia, cachexia and pre-cachexia: joint document elaborated by Special Interest Groups (SIG) 'cachexia-anorexia in chronic wasting diseases' and 'nutrition in geriatrics'. Clin Nutr 29, 154-159.

3. Houston DK, Nicklas BJ \& Ding J (2008) Dietary protein intake is associated with lean mass change in older, communitydwelling adults: the Health, Aging, and Body Composition (Health ABC) Study. Am J Clin Nutr 87, 150-155.

4. Beasely JM, LaCroix AZ, Neuhouser ML, et al. (2010) Protein intake and incident frailty in the Women's Health Initiative Observational Study. J Am Geriatr Soc 58, 1063-1071.

5. Hughes VA, Roubenoff R, Wood M, et al. (2004) Anthropometric assessment of 10-y changes in body composition in the elderly. Am J Clin Nutr 80, 475-482.

6. Dillon EL, Casperson SL, Durham WJ, et al. (2011) Muscle protein metabolism responds similarly to exogenous amino acids in healthy younger and older adults during NO-induced hyperemia. Am J Physiol Regul Integr Comp Physiol 301, R1408-R1417.

7. Timmerman KL, Lee JL, Fujita S, et al. (2010) Pharmacological vasodilation improves insulin-stimulated muscle protein anabolism but not glucose utilization in older adults. Diabetes 59, 2764-2771.

8. Beyer I, Mets T \& Bautmans I (2012) Chronic low-grade inflammation and age-related sarcopenia. Curr Opin Clin Nutr Metab Care 15, 12-22.

9. Stookey JD, Adair LS \& Popkin BM (2005) Do protein and energy intakes explain long-term changes in body composition? J Nutr Health Ageing 9, 5-17.

10. Morris MS \& Jacques PF (2012) Total protein, animal protein and physical activity in relation to muscle mass in middle-aged and older Americans. Br J Nutr 109, 1294-1303.
11. Mitchell D, Haan MN, Steinberg FM, et al. (2003) Body composition in the elderly: the influence of nutritional factors and physical activity. J Nutr Health Ageing 7, 130-139.

12. Baumgartner RN, Waters DL, Gallagher D, et al. (1999) Predictors of skeletal muscle mass in elderly men and women. Mech Ageing Dev 107, 123-136.

13. Volpi E, Campbell WW, Dwyer JT, et al. (2012) Is the optimal level of protein intake for older adults greater than the recommended dietary allowance? J Gerontol A Biol Sci Med Sci 68, 677-681.

14. Bauer J, Biolo G, Cederholm T, et al. (2013) Evidence-based recommendations for optimal dietary protein intake in older people: a position paper from the PROT-AGE Study Group. J Am Med Dir Assoc 14, 542-559.

15. Nordic Council of Ministers (2014) Nordic Nutrition Recommendations 2012. Copenhagen: Nordic Council of Ministers.

16. Yang Y, Breen L, Burd NA, et al. (2012) Resistance exercise enhances myofibrillar protein synthesis with graded intakes of whey protein in older men. BrJ Nutr 108, 1-9.

17. Breen L \& Phillips SM (2011) Skeletal muscle protein metabolism in the elderly: interventions to counteract the 'anabolic resistance' of ageing. Nutr Metab 8, 68-68.

18. Churchward-Venne TA, Burd NA \& Phillips SM (2012) Nutritional regulation of muscle protein synthesis with resistance exercise: strategies to enhance anabolism. Nutr Metab 9, 40.

19. Norton LE, Layman DK, Bunpo P, et al. (2009) The leucine content of a complete meal directs peak activation but not duration of skeletal muscle protein synthesis and mammalian target of rapamycin signaling in rats. J Nutr 139, 1103-1109.

20. Aquilani R, Opasich C, Gualco A, et al. (2008) Adequate energy-protein intake is not enough to improve nutritional and metabolic status in muscle-depleted patients with chronic heart failure. Eur J Heart Fail 10, 1127-1135.

21. Fuller JC, Baier S, Flakoll P, et al. (2011) Vitamin D status affects strength gains in older adults supplemented with a combination of $\beta$-hydroxy- $\beta$-methylbutyrate, arginine, and lysine. J Parenter Enteral Nutr 35, 757-762.

22. Verhoeven S, Vanschoonbeek K, Verdijk LB, et al. (2009) Long-term leucine supplementation does not increase muscle mass or strength in healthy elderly men. Am J Clin Nutr 89, $1468-1475$.

23. Aubertin-Leheudre M \& Adlercreutz H (2009) Relationship between animal protein intake and muscle mass index in healthy women. Br J Nutr 102, 1803-1810.

24. Hartz AJ, Rupley DC \& Rimm AA (1984) The association of girth measurements with disease in 32,856 women. Am J Epidemiol 119, 71-80.

25. Jensen KH \& Jorgensen T (1991) Incidence of gallstones in a Danish population. Gastroenterology 100, 790-794.

26. Heitmann BL (1991) Body fat in the adult Danish population aged 35-65 years: an epidemiological study. Int J Obes 15, 535-545.

27. Heitmann BL (1993) The influence of fatness, weight change, slimming history and other lifestyle variables on diet reporting in Danish men and women aged 35-65 years. Int J Obes 17, 329-336.

28. Ma Y, Olendzki BC, Pagoto SL, et al. (2010) What are patients actually eating: the dietary practices of cardiovascular disease patients. Curr Opin Cardiol 25, 518-521.

29. United States Department of Agriculture (2009) USDA National Nutrient Databank for food composition. http:// www.ars.usda.gov/research/projects/projects.htm?accn_no= 416473 (accessed 10 December 2013).

30. Robinson MJ, Burd NA, Breen L, et al. (2013) Dose-dependent responses of myofibrillar protein synthesis with beef ingestion are enhanced with resistance exercise in middle-aged men. App Physiol Nutr Metab 38, 120-125. 
31. Breen L \& Phillips SM (2013) Interactions between exercise and nutrition to prevent muscle waste during ageing. Br J Clin Pharmacol 75, 708-715.

32. Koopman R, Verdijk LB, Beelen M, et al. (2008) Co-ingestion of leucine with protein does not further augment post-exercise muscle protein synthesis rates in elderly men. Br J Nutr 99 571-580.

33. Taaffe DR (2006) Sarcopenia: exercise as a treatment strategy. Aust Fam Physician 35, 130-134.

34. Haran P, Rivas D \& Fielding R (2012) Role and potential mechanisms of anabolic resistance in sarcopenia. $J$ Cachexia Sarcopenia Muscle 3, 157-162.

35. Danesh J, Whincup P, Walker M, et al. (2000) Low grade inflammation and coronary heart disease: prospective study and updated meta-analyses. BMJ 321, 199-204.

36. Henderson GC, Nair KS, Dhatariya K, et al. (2009) Higher muscle protein synthesis in women than men across the lifespan, and failure of androgen administration to amend age-related decrements. FASEB J 23, 631-641.
37. Smith GI, Atherton P, Villareal DT, et al. (2008) Differences in muscle protein synthesis and anabolic signaling in the postabsorptive state and in response to food in 65-80 year old men and women. PLOS ONE 3, e1875.

38. Smith GI, Atherton P, Reeds DN, et al. (2009) No major sex differences in muscle protein synthesis rates in the postabsorptive state and during hyperinsulinemiahyperaminoacidemia in middle-aged adults. J Appl Physiol 107, 1308-1315.

39. Heitmann BL \& Frederiksen P (2007) Imprecise methods may both obscure and aggravate a relation between fat and breast cancer. Eur J Clin Nutr 61, 925-927.

40. Heitmann BL (1990) Prediction of body water and fat in adult Danes from measurement of electrical impedance. A validation study. Int J Obes 14, 789-802.

41. Brodie DPHD, Moscrip VMSC \& Hutcheon RMSC (1998) Body composition measurement: a review of hydrodensitometry, anthropometry, and impedance methods. Nutrition 14, 296-310. 